\title{
Glomus tumour of the hallux: diagnosis by Doppler-shift ultrasound and digital subtraction angiography
}

\author{
Louis Kreel, Anna Thornton and Bruce J. Pardy ${ }^{1}$ \\ Departments of Radiology and ${ }^{1}$ Surgery, Newham General Hospital, Glen Road, London E13 8RU, UK
}

\begin{abstract}
Summary: A case is presented of a glomangioma with typical history and clinical findings, proven by operation and histology. Unique radiographic features are demonstrated including visualization of the tumour on a soft tissue radiograph and associated hyperaemic bone changes, continuous wave Doppler results indicating hyperaemia and an arterio-venous malformation, and the clear demonstration of the tumour in both frontal and lateral views was possible by intra-arterial digital subtraction angiography (DSA) under local anaesthesia. Fibrous dysplasia of a femur was an incidental finding.
\end{abstract}

\section{Introduction}

Patients with extremity pain caused by a glomus tumour frequently remain undiagnosed for many years, the mean interval between onset of symptoms and treatment in one series being seven years (Carroll $\&$ Berman, 1972). When the tumour is pigmented and subungual, diagnosis is straightforward providing the possibility of a glomus tumour is considered. When the tumour is deep on the palmar or plantar surface of the digit the lesion is not visible, but the plain films may show a small soft tissue mass and erosion of the adjacent phalanx (Carroll \& Berman, 1972; Seim \& Van Demark, 1970). Conventional arteriography can demonstrate characteristic features of a glomus tumour (Trignano et al., 1981), usually requiring arterial catheterization under general anaesthesia (Carroll \& Berman, 1972; Seim \& Van Demark, 1970; Trignano et al., 1981; Mercier et al., 1970) and films of adequate quality are not always obtained. Use of newer techniques of investigation to show limb blood flow characteristics and arterial anatomy are less invasive and can provide characteristic appearances (Trignano et al., 1981). We report the case of a 17 year old girl with increasing left foot pain over a period of eight years, in whom the diagnosis of glomus tumour on the plantar surface of the hallux was made using the new techniques, thus enabling complete surgical excision and total relief of pain.

Correspondence: L. Kreel, M.D., F.R.C.P., F.R.C.R. Accepted: 3 October 1985

\section{Case report}

A girl aged 17 years presented with an 8-year history of progressive and constant pain in her left foot, aggravated by heat but not by cold. The symptoms were said to date from an episode of moderate trauma to the toe by a supermarket trolley. The pain caused difficulty with sleeping at night, depression, a marked limp, and interference with her work and social life, particularly her hobby as a drum majorette. After numerous medical consultations over a 6-year period including neurological and psychological assessments which suggested neurotic behaviour, the patient presented for surgical opinion at our institution.

The left foot appeared quite normal, but was exquisitely tender, particularly over the distal phalanx of the great toe. The only other finding was that the whole foot was obviously warm compared with the other extremities. There was no evidence of arterial or venous disorder, or of neuropathy.

A plain film of the feet showed marked osteopaenia of the left big toe, first metatarsal, and to a lesser extent of the second toe (Figure 1).

Directional Doppler ultrasound with arterial velocity traces (Vasoscan, Sonicaid) showed normal triphasic blood flow in the right popliteal, posterior tibial and dorsalis pedis arteries but there was failure of reversal of blood flow during early diastole in corresponding arteries on the affected side (Figure 2). These findings were interpreted as indicating an arterio-venous shunt in the foot, and furthermore the 
isotope bone scan showed increased uptake in the left tarsus and hallux (Figure 3).

Arteriography was requested but control films showed a lytic lesion in the proximal diaphysis of the femur considered to have the features of a large area of fibrous dysplasia, confirmed by bone biopsy (Figure 4).

Digital subtraction angiography (DSA) of the first toe with a venous pressure pump injection failed to show any abnormality and was therefore followed by

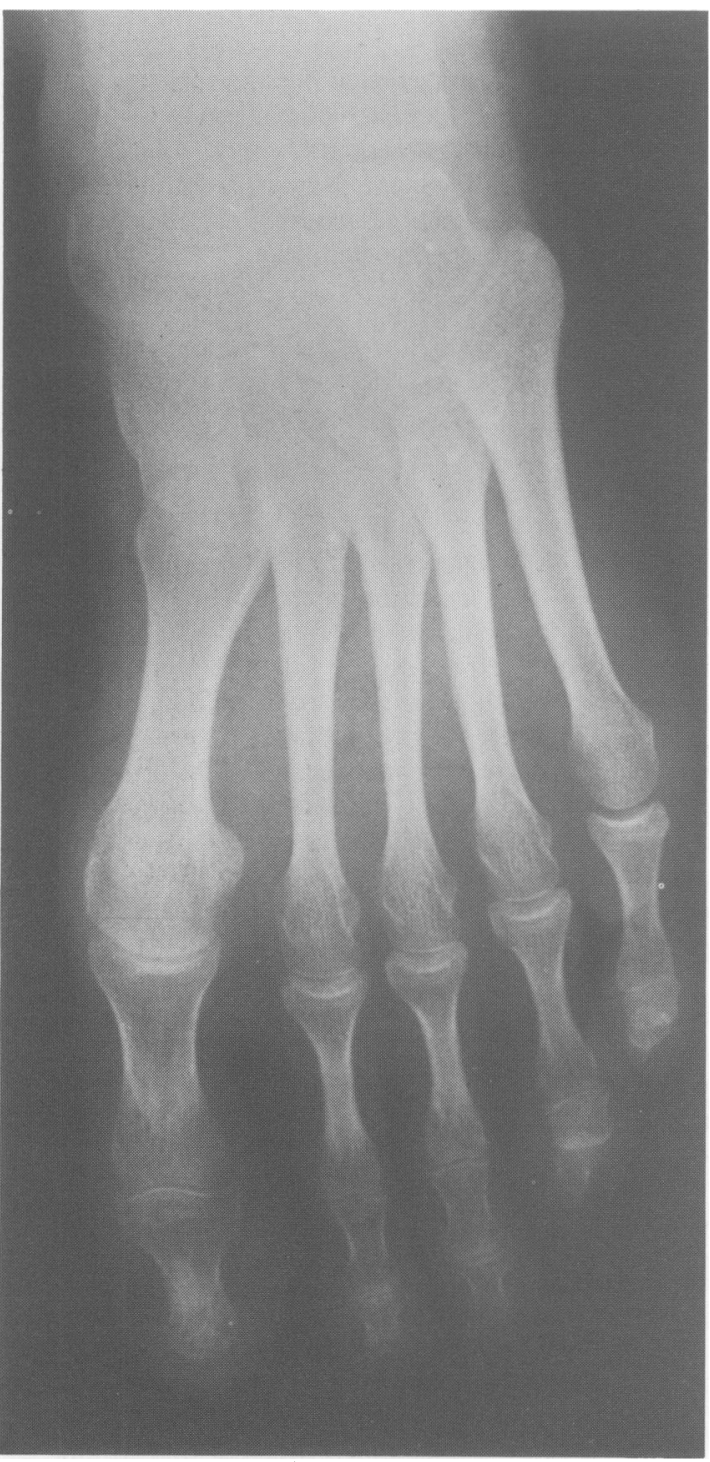

Figure 1 Marked periarticular osteopaenia particularly of the hallux.
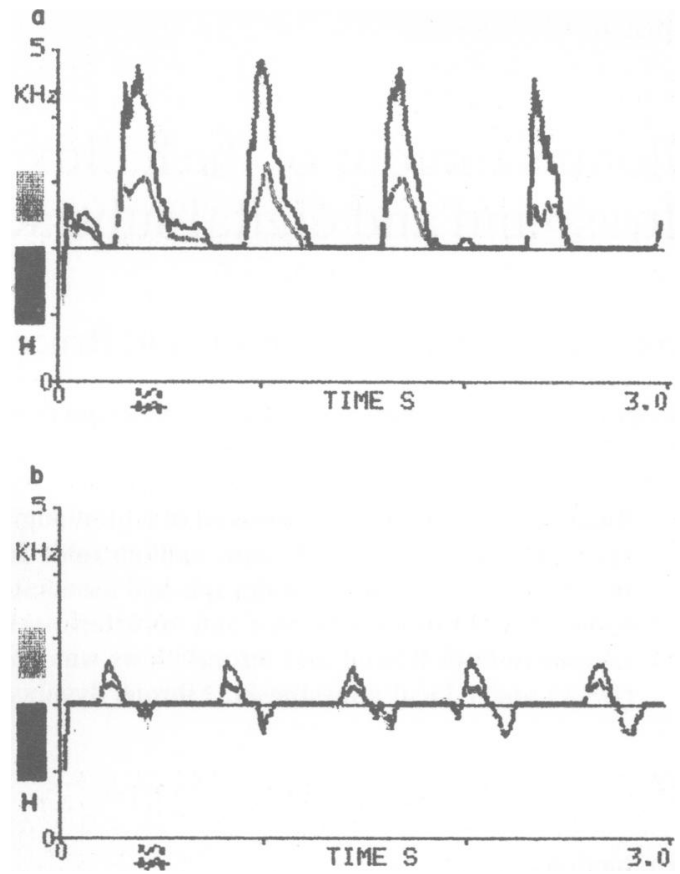

Figure 2 (a) Directional Doppler ultrasound with arterial velocity tracings of the left anterior tibial artery showing high systolic peak on a narrow base with absent reversed flow. (b) Right anterior tibial artery (normal side) for comparison.

an arterial injection via a small catheter in the common iliac artery. The procedure was performed under local anaesthetic (lignocaine) and diazepam $5 \mathrm{mg}$ i.v. using non-ionic contrast medium. Frontal and lateral films clearly demonstrated a one centimetre rounded vascular lesion with a large feeding vessel, localized pooling and rapid venous drainage but required a 'run-off' time of 40 seconds.

The vascular lesion was in the pulp space adjacent to the plantar surface of the terminal phalanx of the big toe with the pathognomonic features of a glomus tumour (Figure 5). Careful inspection of the original plain films of the left foot suggested the presence of a small soft tissue mass in a similar position. Soft tissue films were therefore taken of the big toe and on the lateral film a small soft tissue mass could be seen as well as minimal adjacent pressure erosion of bone (Figure 6).

\section{Operative findings, histology and follow up}

Under general anaesthesia, surgical excision of the glomus tumour was achieved under a bloodless field. 
A proximally based flap of hallux skin and subcutaneous tissue was raised off the terminal phalanx, a gelatinous tumour adjacent to bone was removed and the bone curetted. Histological examination of the excised material showed a glomus tumour.

Following operation the foot pain resolved completely, and the directional Doppler arterial velocity traces returned to normal (Figure 7).

\section{Discussion}

The historical and clinical aspects, physiology, pathology and arteriographic features of glomus tumour (glomangioma) are well recognized and documented (Carroll \& Berman, 1972; Seim \& Van Demark, 1970; Trignano et al., 1981; Mercier et al., 1970; Carlstedt \& Lugnegard, 1983) but the role of the newer investigations in this condition have not been described.

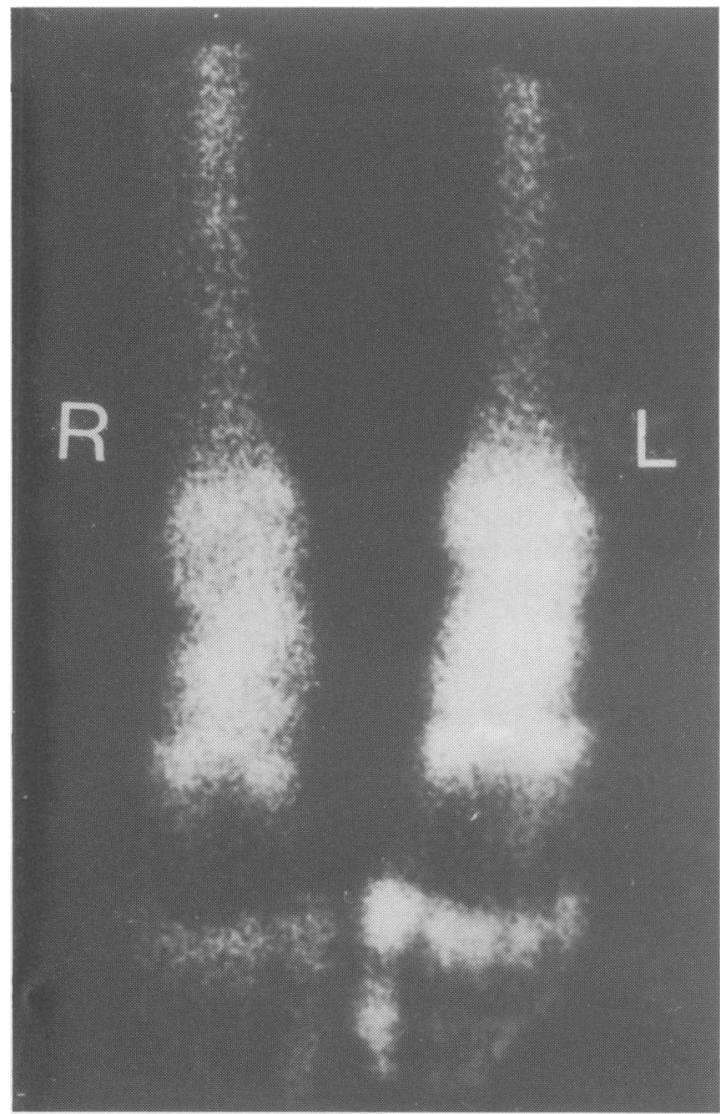

Figure 3 Isotope bone scan of feet demonstrating the marked increased uptake in the tarsus, hallux and metacarpo-phalangeal joints due to marked hyperaemia.

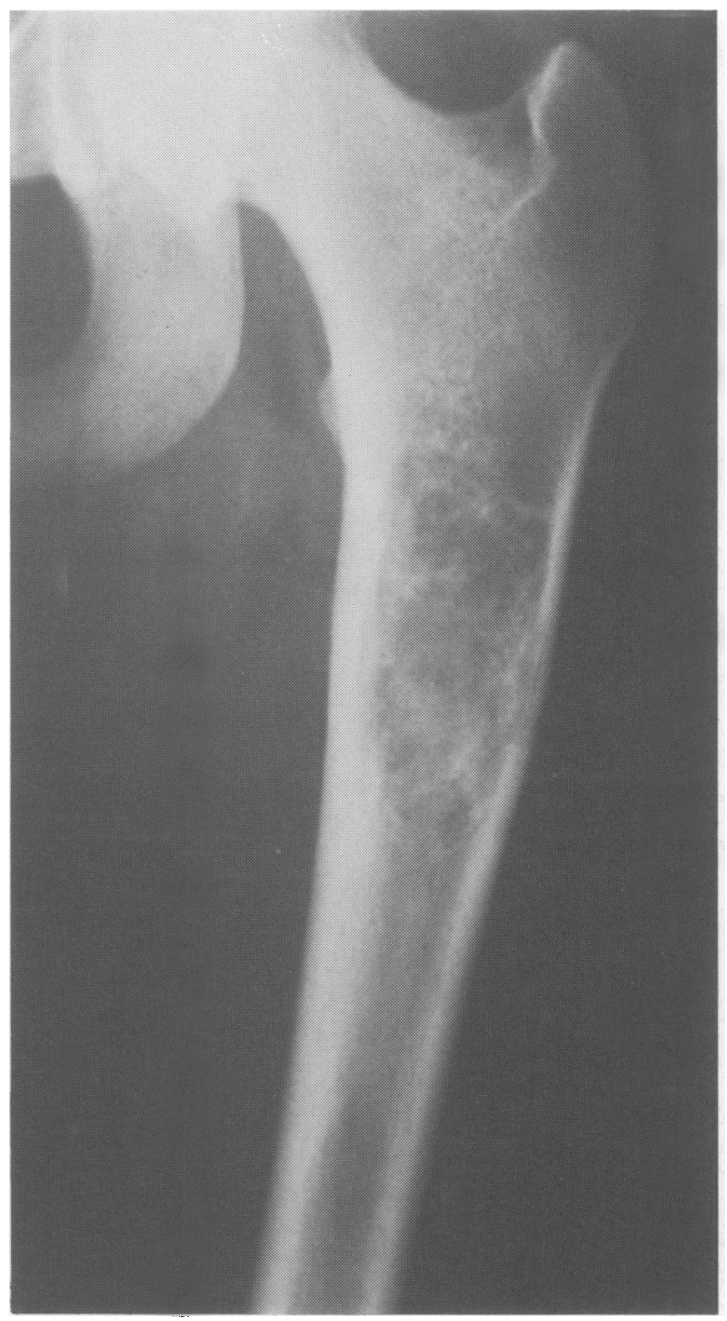

Figure 4 A large area of fibrous dysplasia was noted on pre-contrast angiography films and confirmed by bone biopsy, almost certainly an incidental finding as no similar case can be found in the literature.

Because the diagnosis of glomus tumour is often very delayed, one third being 6 years or more in one series (Carlstedt et al., 1983), use of these newer techniques should be of value in promoting prompt treatment with relief of pain.

Our patient presented with the classical triad of pain, extreme hyperanaesthesia, and temperature sensitivity, although this patient was more sensitive to heat than cold, and illustrates the marked delay that may occur in diagnosis ultimately bringing the patient's psyche into question.

Most glomus tumours are situated in the terminal 

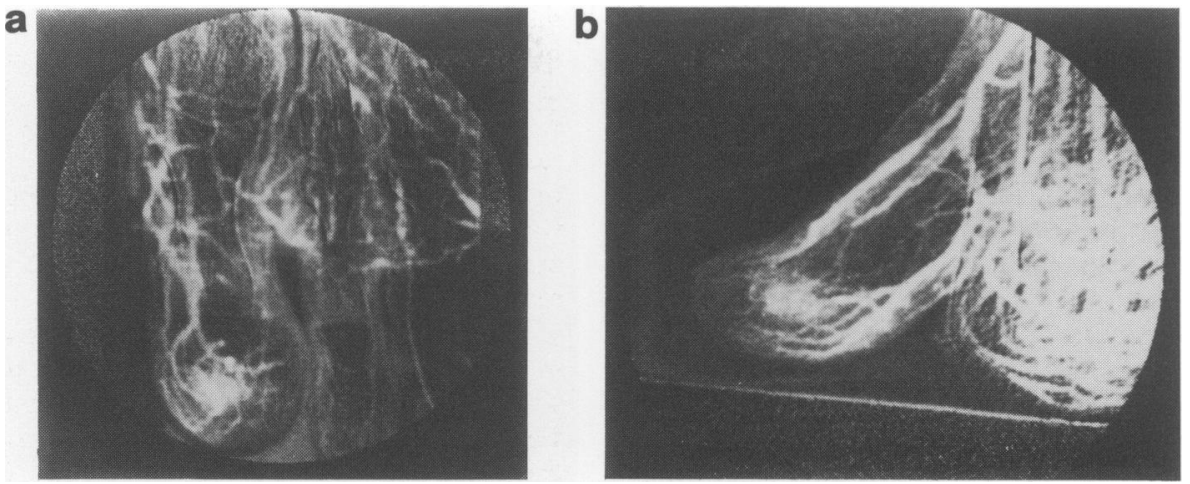

Figure 5 (a) and (b) Digital subtraction angiography showing large feeding digital artery leading to a small area of intense blush and markedly dilated draining veins shown $\mathbf{4 0}$ seconds after contrast injection into the iliac artery but could not be demonstrated by an intravenous injection.

phalanx of a finger, and most are sub-ungual (Carroll \& Berman, 1972; Carlstedt et al., 1983). Other sites are the hand and foot, but reports of glomus tumour in the toe are extremely uncommon, probably because glomus bodies are relatively sparse in the toes (Strahan \& Bailie, 1972).

The glomus tumour in our patient was on the plantar surface of the hallux, was associated with pressure erosion of the adjacent bone and osteopaenia. The glomus tumour could be seen within the soft tissues of the pulp space of the big toe on the lateral view on a soft tissue plain film; but only in retrospect when its position had been clearly visualized after

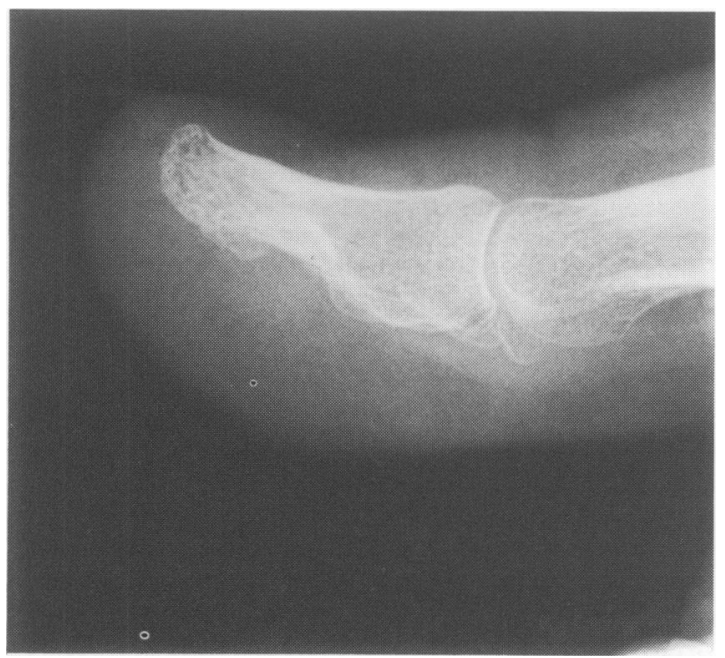

Figure 6 Soft tissue film showing glomus tumour adjacent to pressure deformity on the plantar surface of the distal phalanx corresponding to the tumour as seen on digital subtraction angiography.
DSA. The adjacent bone erosion was also visible. However, at the time, the relevance of these findings was not sufficiently appreciated, and a review of the literature suggested that soft tissue visualization of a glomus tumour has rarely been reported (Carroll \& Berman, 1972; Seim \& Van Demark, 1970; Trignano et al., 1981; Mercier et al., 1970; Carlstedt \& Lugnegard, 1983; Strahan \& Bailie, 1972). The appearances would probably have been seen even more clearly on xerograe̊ phy. The pressure changes on the adjacent bone have been well described, as have the reactive changes, but in this patient there was also a particularly marked localized osteopaenia.

The directional Doppler arterial velocity traces indicated fistula-type blood flow in the affected leg, and it is interesting that such a small lesion produced so marked a change in the arterial wave form. It is possible that the shunt through the glomus tumour was responsible but the general vasodilatation in the left foot probably also contributed to this effect. In this

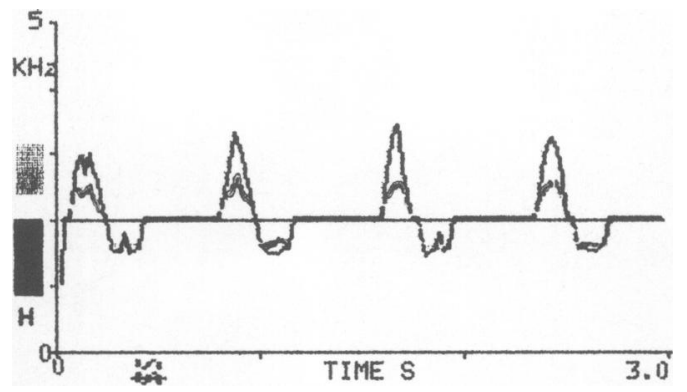

Figure 7 Normal trace of left anterior tibial artery following removal of glomangioma showing diminution of the height of the systolic wave and return of the negative phase. 
case, the findings on plain films together with the changes on directional Doppler examination could be considered pathognomonic; however, angiography was deemed essential before surgical exploration.

The large area of fibrous dysplasia at the upper end of the femur was an incidental finding, there being no previous report of such an association. However, it is just conceivable that these lesions may in the past have been overlooked because there is no particular indication to examine the whole skeleton.

In this patient DSA played a major role in diagnosis. The lesion could not be demonstrated by a transvenous injection in the right atrium, but was well shown by injection of contrast into the ipsilateral common iliac artery. A pressure injector was needed, even though the pressure used was low, but vasodilators were not required. Because of the minimal discomfort associated with use of a low osmolality contrast medium, this examination could be carried out using only diazepam as sedation. The typical angiographic features could be demonstrated in both the frontal and lateral views on DSA which showed the multiple enlarged distal arteries to the big toe, a large single feeding vessel, the prolonged blush of the tumour and the marked venous filling. A major advantage of DSA was the ease with which the diagnostic phase of the examination was obtained considering that it was $\mathbf{4 0}$ seconds after the injection.

The investigation of prolonged severe digital pain must include consideration of a glomus tumour, and when no lesion is visible, soft tissue frontal and lateral views should be taken. Directional arterial Doppler traces may show fistula-type blood flow in the limb, and DSA using a selective intra-arterial injection of low osmolality contrast medium can give information not only of diagnostic importance, but which also accurately localizes the tumour for complete removal by surgery.

\section{References}

CARLSTEDT, T. \& LUGNEGARD, H. (1983). Glomus tumor in the hand; A clinical and morphological study. Acta Orthopaedica Scandinavica, 54, 296.

CARROLL, R.E. \& BERMAN, A.T. (1972). Glomus tumors of the hand. Journal of Bone and Joint Surgery, 54-A, 691.

GERMAIN, H., MERCIER, H.G.R., VANNEUVILLE, G., BRESSON, P., PATOUILlARD, P. \& LAGARDE, R. (1970). A propos d'un nouveau cas d'une tumeur glomique sousunguéale artériographiée. Journal de Chirurgie (Paris), 99, 541 .
SEIM, H.C. \& VAN DEMARK, R.E. (1970). Painful forefoot from glomus tumor. South Dakota Journal of Medicine, 23, 23.

STRAHAN, J. \& BAILIE, H.W.C. (1972). Glomus tumour - A review of 15 clinical cases. British Journal of Surgery, 59, 91.

TRIGNANO, M., SORO, P., SPISSU, M. \& CASOLO, P. (1981). Le Doppler dans le diagnostic des glomus. Journal des Maladies Vasculaires (Paris), 6, 161. 